\title{
Article \\ OtoPair: Combining Right and Left Eardrum Otoscopy Images to Improve the Accuracy of Automated Image Analysis
}

\author{
Seda Camalan ${ }^{1, * \mathbb{C}}$, Aaron C. Moberly ${ }^{2}$, Theodoros Teknos ${ }^{3}$, Garth Essig ${ }^{2}$, Charles Elmaraghy ${ }^{2}$, \\ Nazhat Taj-Schaal ${ }^{4}$ and Metin N. Gurcan ${ }^{1}$ (D) \\ 1 Center for Biomedical Informatics, Wake Forest School of Medicine, Winston-Salem, NC 27101, USA; \\ mgurcan@wakehealth.edu \\ 2 Department of Otolaryngology, Ohio State University, Columbus, OH 43212, USA; \\ Aaron.Moberly@osumc.edu (A.C.M.); garth.essig2@osumc.edu (G.E.); \\ Charles.Elmaraghy@nationwidechildrens.org (C.E.) \\ 3 University Hospitals Seidman Cancer Center, Cleveland, OH 44106, USA; theodoros.teknos@osumc.edu \\ 4 Department of Internal Medicine, Ohio State University, Columbus, OH 43210, USA; \\ Nazhat.Taj-schaal@osumc.edu \\ * Correspondence: scamalan@wakehealth.edu; Tel.: +1-(336)-713-7675
}

Citation: Camalan, S.; Moberly, A.C.; Teknos, T.; Essig, G.; Elmaraghy, C.;

Taj-Schaal, N.; Gurcan, M.N. OtoPair: Combining Right and Left Eardrum Otoscopy Images to Improve the Accuracy of Automated Image Analysis. Appl. Sci. 2021, 11, 1831. https://doi.org/10.3390/app11041831

Academic Editor: Marcos

Ortega Hortas

Received: 31 December 2020

Accepted: 16 February 2021

Published: 19 February 2021

Publisher's Note: MDPI stays neutral with regard to jurisdictional claims in published maps and institutional affiliations.

Copyright: (c) 2021 by the authors. Licensee MDPI, Basel, Switzerland. This article is an open access article distributed under the terms and conditions of the Creative Commons Attribution (CC BY) license (https:/ / creativecommons.org/licenses/by/ $4.0 /)$.

\begin{abstract}
The accurate diagnosis of otitis media (OM) and other middle ear and eardrum abnormalities is difficult, even for experienced otologists. In our earlier studies, we developed computer-aided diagnosis systems to improve the diagnostic accuracy. In this study, we investigate a novel approach, called OtoPair, which uses paired eardrum images together rather than using a single eardrum image to classify them as 'normal' or 'abnormal'. This also mimics the way that otologists evaluate ears, because they diagnose eardrum abnormalities by examining both ears. Our approach creates a new feature vector, which is formed with extracted features from a pair of high-resolution otoscope images or images that are captured by digital video-otoscopes. The feature vector has two parts. The first part consists of lookup table-based values created by using deep learning techniques reported in our previous OtoMatch content-based image retrieval system. The second part consists of handcrafted features that are created by recording registration errors between paired eardrums, color-based features, such as histogram of $a^{*}$ and $b^{*}$ component of the $L^{*} a^{*} b^{*}$ color space, and statistical measurements of these color channels. The extracted features are concatenated to form a single feature vector, which is then classified by a tree bagger classifier. A total of 150-pair (300-single) of eardrum images, which are either the same category (normal-normal and abnormal-abnormal) or different category (normal-abnormal and abnormal-normal) pairs, are used to perform several experiments. The proposed approach increases the accuracy from $78.7 \%( \pm 0.1 \%)$ to $85.8 \%( \pm 0.2 \%)$ on a three-fold cross-validation method. These are promising results with a limited number of eardrum pairs to demonstrate the feasibility of using a pair of eardrum images instead of single eardrum images to improve the diagnostic accuracy.
\end{abstract}

Keywords: acute otitis media; eardrum classification; otoscopy; transfer learning

\section{Introduction}

Ear diseases are one of the diseases that can easily be treated when diagnosed at the right time and when appropriate treatment methods are applied. Otherwise, ear diseases may cause hearing loss or other complications. An otoscopic examination is one of the most basic and common tools used to examine the ear canal and eardrum (tympanic membrane, TM) [1,2]. With the help of otoscopic examination and clinical features, the perforation of TM, cholesteatoma, acute otitis media (AOM), and chronic otitis media (COM) can be diagnosed by otolaryngologists and pediatricians. However, an examination by an experienced and skillful physician may not always be possible. Computer-aided diagnosis (CAD) systems may be useful to help physicians who lack the same otoscopy experience [3]. 
Most CAD systems use a classification method to determine whether the middle ear has an infection [4-8], because otitis media (OM) is one of the most common diseases for children under the age of five years $[9,10]$. Other ear diseases, such as retraction, perforation, and tympanosclerosis are classified as abnormal eardrum images against normal eardrum images by the automated CAD system [11-13]. In addition to classification methods, there are also approaches to segment and classify the TM on otoscopic images [14-16]. Recently, composite images, which are created by selecting certain otoscopy video frames and stitching them together, are also used to increase the probability of detecting ear pathology $[17,18]$. Our previous study using OtoMatch, a content-based image retrieval (CBIR) system, is also a good example of a CAD system that is designed to help physicians [19].

CAD approaches for TM analysis, which are used to classify and/or segment the eardrum, can be collected under two categories: hand-crafted and deep learning-based. For a hand-crafted approach, the most commonly used features are color-based information in addition to traditional texture approaches $[5,12,14,15,20,21]$. The color-based information has been common, because there are significant differences between normal and abnormal cases of eardrums. The deep learning-based approach is also used more than the texturebased approach, because it is typically more accurate $[4,22]$. One study has used both a hand-crafted and deep learning-based approach to classify otoscopy images [7].

CAD for OM abnormalities has only been applied to single TM images, to the best of our knowledge. Lee et al. proposed a convolutional neural network (CNN)-based approach that detects the ear's side, but this information was not used to classify paired images (right and left ears) together [23]. However, physicians typically examine both ears during a physical exam before making a diagnosis. In this first study to classify a pair of eardrum images together, we propose a system called OtoPair that uses deep learning- and color-based features to classify a pair of TM images as 'normal-normal', 'normal-abnormal', 'abnormal-normal', or 'abnormal-abnormal'. A lookup table was created to extract deep learning-based features. The pre-processing steps for creating the lookup table are similar to our previous study, called OtoMatch [19]. The lookup table values of the paired images were analyzed according to their labels to determine the association between right and left ear values. Additionally, we investigated the contribution of color-based features to the classification accuracy.

\section{Materials and Methods}

Our pairwise classification system, OtoPair, has the following components: preprocessing and data augmentation, feature extraction to generate a lookup table, feature vector formation for a pair of eardrum images, and normal/abnormal classification for the pairs (see Figure 1).

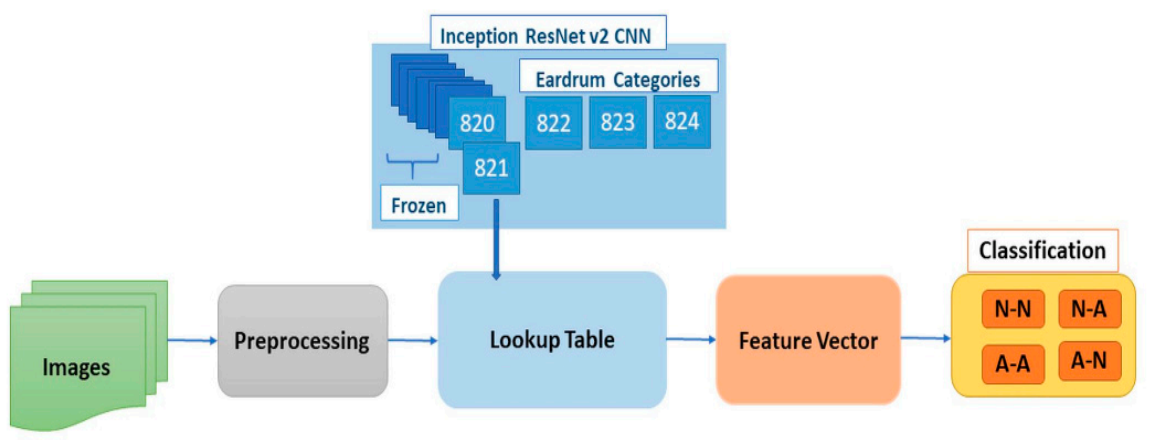

Figure 1. Block Diagram of OtoPair System. Dataset: Train and Validation images, Preprocessing: remove black margin and time text, Lookup Table: generated using Inception ResNet v2 (first 819 layers are frozen, last five layers trained, the values taken from the layer 821st and last three layers updated for eardrum categories), Feature Vector: generated by using pair eardrum images lookup table values, Classification: classify the pair of eardrum images (N-N: Normal-Normal, N-A: Normal-Abnormal, A-A: Abnormal-Abnormal, A-N: Abnormal-Normal). 
The pre-processing steps remove the black margin and date/time text on the images, as explained in our previous study, OtoMatch [19]. The feature extraction is completed in two steps: transfer learning-based and handcrafted. In order to obtain transfer learningbased lookup table features, we used the infrastructure of our previous work [19]. Finally, the paired images were classified, and the performance of the system was evaluated by 10 -fold validation.

\subsection{Dataset}

All of the images used in this study are captured from adult (174 ears) and pediatric (124 ears) patients at primary care clinics and Ear, Nose, and Throat (ENT) facilities of the Ohio State University (OSU) and Nationwide Children's Hospital (NCH) in Columbus, Ohio, US with the IRB approval (Study Number: 2016H0011). The images from adult patients were collected in clinic by a board-certified ENT physician with fellowship training in neurotology (i.e., a clinical focus on ear disease); images from pediatric patients were collected in the operating room at the time of tympanostomy tube placement by a boardcertified ENT physician with fellowship training in pediatric otolaryngology. Additionally, conforming to the rules set by the Ohio State University Institutional Review Board, all of the samples were fully anonymized while creating the experimental dataset.

In this study, a total of 150-pair (i.e., 300 individual) eardrum images were used to train and test the system, with images being included if a complete image pair was available (i.e., images from both ears were available and had sufficient focus and lighting to evaluate the eardrum), and were diagnosed as normal, middle ear effusion, or tympanostomy tube present. Each pair consists of the right and left ear images of the same person that were captured in the same visit. Table 1 shows the number of images for each category (normal-abnormal). We only included two categories of abnormality: effusion (fluid) of the middle ear and tympanostomy tube because there was not a sufficient number of images in other categories to train and test our classifiers properly. Again, for the same reason, we investigated the problem of normal-abnormal pair classification, as opposed to classifying the pairs according to the type of abnormality separately. In addition to the number of pair images, 137 single images (83-Abnormal, 54-Normal) were used to validate the developed system's deep learning part while extracting the lookup table features.

Table 1. The number of images for each class of eardrum types. Both right and left eardrum images have the same diagnosis, and right and left different category image numbers in the dataset.

\begin{tabular}{ccccc}
\hline Category & $\begin{array}{c}\text { Number of } \\
\text { Single Images }\end{array}$ & $\begin{array}{c}\text { Number of the } \\
\text { Same Category } \\
\text { Pair Images }\end{array}$ & $\begin{array}{c}\text { Number of the } \\
\text { Right Images }\end{array}$ & $\begin{array}{c}\text { Number of the } \\
\text { Left Images }\end{array}$ \\
\hline $\begin{array}{c}\text { Normal } \\
\text { Abnormal }\end{array}$ & 168 & 71 & 14 & 12 \\
$\begin{array}{c}\text { (Effusion-Tube) } \\
\text { Total }\end{array}$ & 132 & 53 & 12 & 14 \\
\hline
\end{tabular}

Figure 2 shows paired examples from normal, abnormal with effusion, and abnormal with tympanostomy tube categories, demonstrating the variability among the images in different categories. The images from some categories are difficult to distinguish from the others for an untrained person. In many cases, the similarities between the right and left ear are not obvious. The tympanostomy tube abnormality appears differently for the same patient, as can be seen in Figure 2e,f. 


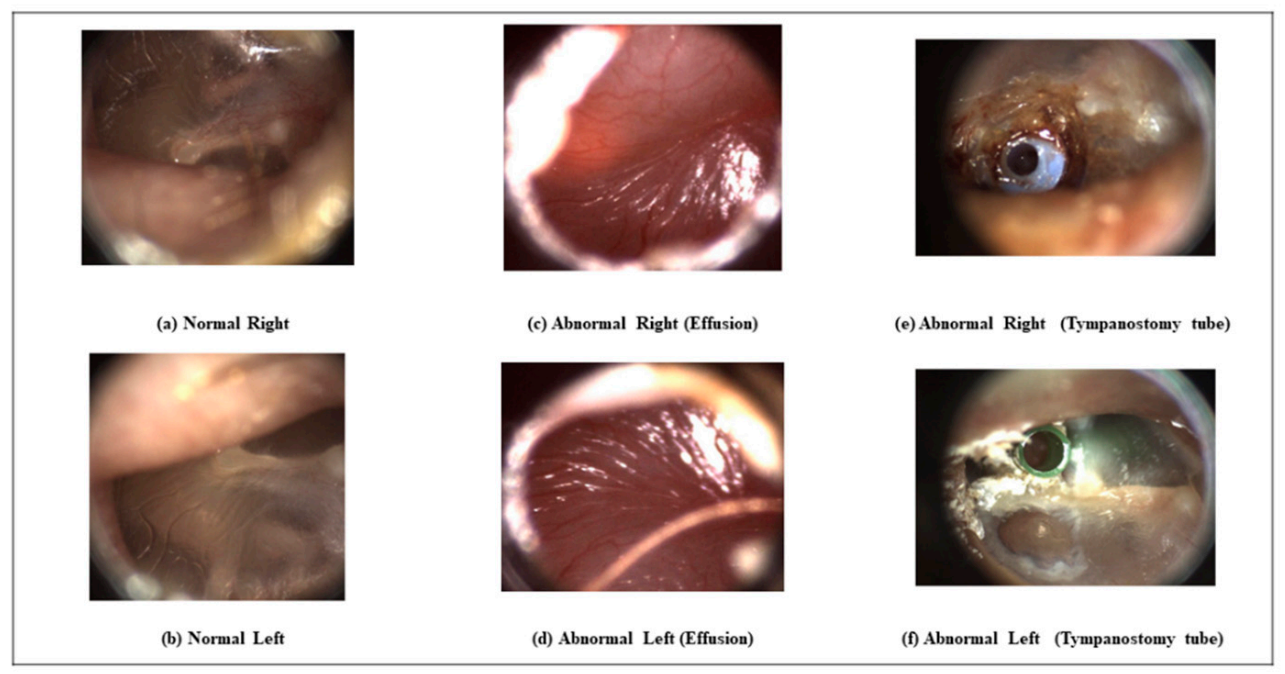

Figure 2. Preprocessed image example from each category: (a) Normal Right, (b) Normal Left, (c) Abnormal Right (Effusion) (d) Abnormal Left (Effusion), (e) Abnormal Right (Tympanostomy tube), and (f) Abnormal Left (Tympanostomy tube).

We used all of the paired image datasets because increasing the number of paired images was critical in this study. The database contained images that were captured in the JPEG format. Additionally, we selected individual images from the frames of otoscopy video clips. Both single images and video frames were the same size (1440 by 1080 pixels) and resolution. Some of the images in the video frames were unfocused, contained large amounts of wax, or did not have the proper illumination. Therefore, we manually selected the best single images and frames to form the appropriate pair of images.

\subsection{Data Augmentation and Preprocessing}

A data augmentation approach was used to increase the number and diversity of images for transfer learning. The augmentation approached included reflecting images both horizontally and vertically, scaling images in the range of 0.7 to 2 , randomly rotating images, shearing images both horizontally and vertically within a range of 0 to 45 degrees, and then translating them within a pixel range from -30 to 30 pixels in both the horizontal and vertical directions.

Regions of interest (RoI) were extracted in the preprocessing step, which is the same as our previous study OtoMatch, in order to extract features from these images [19]. The preprocessing step included reducing the black margin and embedded text (to mark the capture date and time), which may negatively affect the features.

\subsection{Forming Feature Vector}

In order to classify the eardrum pairs as normal or abnormal, the feature extraction was completed in two steps: transfer learning-based lookup table feature extraction and handcrafted feature extraction. The transfer learning-based lookup table features are the same as in our previous work [19] for the eardrum image retrieval system. In this study, they were integrated into the pairwise classification system (Section 2.4). The handcrafted features included registration error, histogram, and statistical measurements of the $a^{*}$ and $b^{*}$ components of the $L^{*} a^{*} b^{*}$ color space.

\subsubsection{Transfer Learning based Feature Extraction}

In our previous work [19], Inception-ResNet-V2 Convolutional Neural Network (CNN) was used. It was pre-trained and validated with 50,000 images set to classify 1000 object categories and learned rich feature representations with 825 layers. A huge number of images is required to re-train the whole network. The first 820 layers were frozen to limit the number of parameters required to retrain the network to avoid overfitting with 
our limited dataset. The last three layers (prediction, softmax, and classification) of the pre-trained network were retrained with otoscope images in our database.

After retraining the network, the resulting features were subjected to pooling that mapped each image into a vector of 1536 features. We relied on the fully connected layer's output, which produced $1 \times 3$ vectors for each training and test image, where three represents the number of image categories in our database: normal, effusion, and tympanostomy tube. The features formed a $1536 \times 1$ vector at the output of the average pooling layer. Therefore, the weights were a $1536 \times 3$ matrix of the fully connected layer. When the transpose of the feature vector was multiplied by the weight vector, it produced a $1 \times 3$ vector, which was established for each of the training set of images. When these vectors were turned to rows of a matrix (size of the number of training images $\times 3$ ), this constitutes the lookup table.

This procedure was applied to a pair of eardrum images for normal/abnormal feature extraction. Inception-ResNet-V2 pre-trained $\mathrm{CNN}$ was used by freezing the first 820 layers. In this study, the number of categories was two (i.e., normal, abnormal), and the weights constitute a $1536 \times 2$ matrix of the fully connected layer. The generated lookup table is a vector of length $N \times 2$, where $N$ is the number of training images. Additionally, test images have a $1 \times 2$ vector after multiplying them by weights. For each pair of the eardrum images, these lookup values are calculated, and a new feature vector is formed using these values.

The steps to create a lookup table from transfer learning can be generalized, as follows:

- Form a feature vector $f_{i}$ as the average pooling layer output for each image, $i$. Its size is $(F \times 1)(F=1536$ in this case).

- $\quad$ Let $w$, of size $(F \times C)$, be the weights of the fully connected layer, where $C$ is the number of training classes $(C=2$ in this case because of two categories: 'normal' and 'abnormal').

- The lookup table values $l_{i}$ for one image, $i$, can be calculated as $l_{i}=f_{i}^{T} \times w$, and its size is $(1 \times C)$.

- If $N$ is the number of images in the dataset (both for training and testing), the lookup table $L_{T}$ is a matrix calculated as concatenation of the lookup table values $l_{i}$ for each image, $i$ and with a size $N \times C(300 \times 2$ for this case $)$.

The lookup table values of the right eardrum image are $R_{1}$ and $R_{2}$, and of the left eardrum image are $L_{1}$ and $L_{2}$. Their ratio $\left(R_{1} / L_{1}, R_{2} / L_{2}\right)$, summation $\left(R_{1}+L_{1}, R_{2}+L_{2}\right)$, and difference $\left(R_{1}-L_{1}, R_{2}-L_{2}\right)$ are also concatenated to form a feature vector. This new vector, which contains both eardrum pairs' features, enables us to classify the pair together by combining the derivative of lookup table values for a pair of eardrum images.

\subsubsection{Handcrafted Feature Extraction}

In addition to the lookup table-based features, we also used handcrafted features, which captured the registration errors between the pair eardrum images. The registration is used to match and compare two or more images that were obtained at different times from different sensors or different viewpoints to find the best transformation that portrays good spatial correspondence among them [24,25]. Image registration is frequently used in medicine to align images from different medical modalities for diagnosis, treatment monitoring, surgery simulation, radiation therapy, assisted/guided surgery, and image subtraction for contrast-enhanced images [26-29]. In our study, we used image registration to calculate the error between the pair of eardrum images and use it as a feature to classify pairs together.

Eardrum image registration is challenging, even for normal cases, because the malleus is positioned differently in the eardrum images of the right and left sides of the same person. Furthermore, the pair images are rarely symmetric, nor are they obtained from the same perspective when captured with an otoscope. For diseased eardrums, registration is more challenging than that for normal cases, because some diseases (e.g., effusion) lead to changes in the eardrum shape and cannot be easily detected with 2D images. 
We used both rigid and non-rigid registration. For both types of registration, there should be moving and target images; moving (source) images transform spatially to align with the target (fixed, sensed) image. Rigid registration [30] includes the translation, scaling, and rotation of the moving image to the target image, and non-rigid matching is done using the demons method [31], which transforms the points, depending on Maxwell's demons and matches the deformed parts of the image. The basis of demon registration forces finds small deformations in temporal image sequences by calculating the optical flow equations. Therefore, the Thirion method estimates the displacement [31] for corresponding match points. Gaussian smoothing is used for displacement for regularization because the demons equation approximates the local displacement in each iteration.

Before registration, each image is converted from color (RGB) to a gray-scale image, and the registration is applied to the gray-scale images. For rigid registration, mutual information is used as the similarity metric. For optimization, a one-plus-one evolutionary optimization algorithm $[32,33]$, which iterates the set of parameters to produce the best possible registration, is used with the initial radius's parameter is 0.009 , epsilon is $1.5 \times 10^{-4}$, the growing factor is 1.01, and a maximum of 300 iterations. After rigid registration, nonrigid demon algorithm image registration [34] with a single modality parameter is applied to a rigid registered image.

The mean square error between the fixed image and registration images is computed as the difference of corresponding pixels and taking the mean square of them and used as a similarity metric between the fixed and moving images. One of the mean square errors is computed after rigid registration, and another one is after non-rigid registration. These two mean square errors concatenated to feature vector starts with lookup table based values.

Lastly, we used $a^{*}$ and $b^{*}$ components of the $L^{*} a^{*} b^{*}$ color space of each pair of eardrum images to extract color-based features to accurately classify the pairs. The $L^{*} a^{*} b^{*}$ color space is the uniform color space with equal distances on the $\mathrm{x}$, $\mathrm{y}$ chromaticity diagram corresponds to equal perceived color difference. In this color space, $\mathrm{L}^{*}$ indicates lightness, and $\mathrm{a}^{*}$ and $\mathrm{b}^{*}$ are the chromaticity coordinates, where $+\mathrm{a}^{*}$ is the red direction, $-\mathrm{a}^{*}$ is the green direction, $+b^{*}$ is the yellow direction, and $-b^{*}$ is the blue direction. The RGB color images are converted to the $\mathrm{L}^{*} \mathrm{a}^{*} \mathrm{~b}^{*}$ color space, and the histogram and statistical measurements of the $a^{*}$ and $b^{*}$ bands are calculated. The histogram of color bands is divided into ten bins, and the number of each bin is concatenated to the feature vector for each pair of images. There are 40 histogram values, which come from two images (right and left images) and two bands ( $a^{*}$ and $b^{*}$ bands) for each ear pair. In addition to the histogram values, statistical measurements of mean, standard deviation, skewness, and kurtosis of each band of pairs. A total of 16 features come from these four statistical measurements. Figure 3 shows a graphical summary of the new feature vector formation.

\subsection{Classification}

After the feature vector of the pair (right and left) eardrum images was formed by concatenating the lookup table values with handcrafted features, these are classified. In order to classify a pair of images together, we aimed to collect all of the features in one vector for each pair. Thus, we could analyze the difference between classifying single images and classifying pairs as 'Normal-Normal' (N-N), 'Abnormal-Abnormal' (A-A), 'NormalAbnormal' (N-A), and 'Abnormal-Normal' (A-N). Single image classification results were obtained after retraining the Inception ResNet-v2 pre-trained network by changing the last three layers according to 'Normal/Abnormal' classes of eardrum images and frozen the first 820 layers to avoid an overfitting of the network. The number of frozen layers was empirically determined from our previous study OtoMatch as 820. For the paired image classification, a newly created feature vector is used in the Tree Bagger algorithm. 


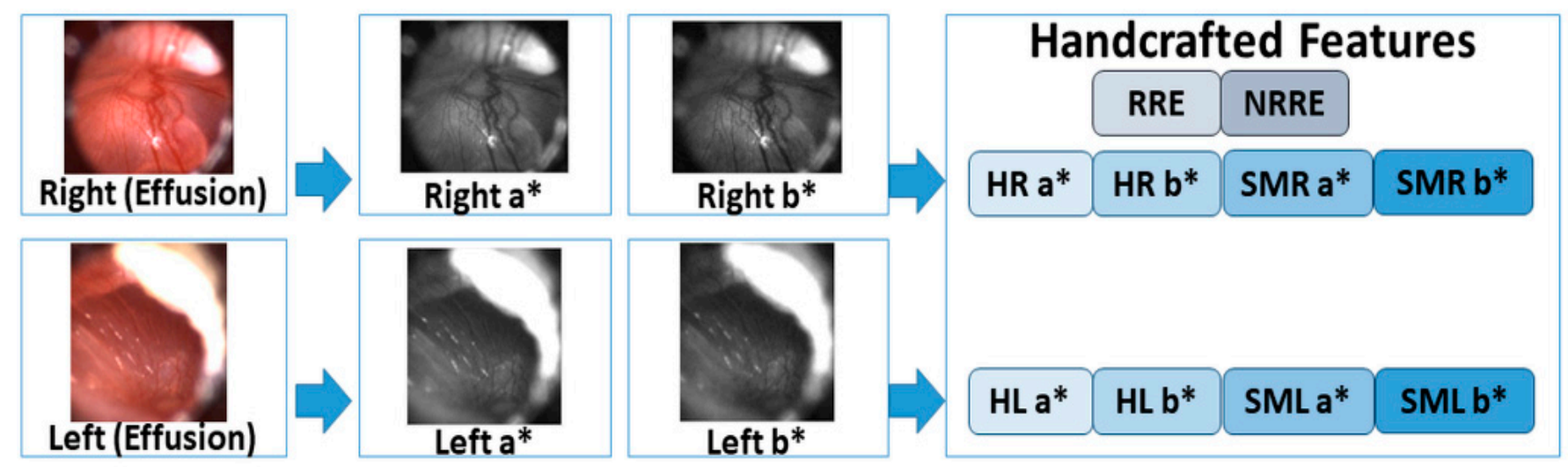

Figure 3. Creation of a feature vector structure for a pair of eardrum images. Rigid and Non-Rigid registration errors were calculated for a pair of eardrums. RGB pair images are converted to the $\mathrm{L}^{*} \mathrm{a}^{*} \mathrm{~b}^{*}$ color space, and $\mathrm{a}^{*}$ and $\mathrm{b}^{*}$ components are used for creating color histograms and calculating statistical measurements. All features are concatenated to form a handcrafted feature vector. RRE: Rigid Registration Error, NRRE: Non-Rigid Registration Error, HR a*: Histogram of Right $a^{*}$, HR $b^{*}$ : Histogram of Right $b^{*}$, HL $a^{*}$ : Histogram of Left $a^{*}$, HL $b^{*}$ : Histogram of Left $b^{*}$, SMR $a^{*}$ : Statistical Measurements of Right $a^{*}$, SMR $b^{*}$ : Statistical Measurements of Right $b^{*}$, SML $a^{*}$ : Statistical Measurements of Left $a^{*}$, SML b*: Statistical Measurements of Left $b^{*}$.

The Tree Bagger algorithm is the ensemble model of bootstrap aggregated decision trees. Multiple decision trees constitute resampling training data with replacement again and again, and voting the trees for majority prediction [35]. These decision trees are the classification trees whose leaves represent class labels; branches represent conjunctions of features that convey to these class labels. In our problem, the leaves are the 'Normal' and 'Abnormal' class labels, and the branches are the conjunction of the feature vector. The out-of-bag (OOB) error method [36] was utilized to measure the prediction error of boosted decision trees models to sub-sample data to train the method. The OOB error is measured by excluding a sub-sample from the training data and calculating the mean prediction error in the bootstrap sample [37]. Subsampling improves the prediction performance by evaluating predictions on observations that are not used in building the tree (defined out-of-bag).

This study modeled the decision tree using the TreeBagger supervised machine learning function in Matlab 2019b software. Selected trees, where the observation is out of the bag, compose the class posterior probabilities' weighted mean. Accordingly, the predicted class is the largest weighted mean of a corresponding class. This is also designed to improve the model's stability and accuracy by reducing the variance without raising the bias. The optimal number of trees decided according to the out-of-bag error changes with the accumulation of trees. In our study, the number of classes (normal and abnormal) and the number of observations (150 pair eardrum image) limit the number of decision trees, which is empirically selected to be five.

\subsection{Experimental Setup}

We selected 150-pair images (see Table 1) from normal and abnormal (effusion and tympanostomy tube) categories, which have the highest number of paired images in our database. Even after we enhanced our database with selected video frames of the videos, our dataset contained 150 paired eardrum images to train the model. Even though we know that the balanced amount of data for each category would avoid the bias towards the majority classes and minimize the overall error rate, we could not add more normalabnormal pair images to our dataset because of the limited number of cases.

Our limited number of pair images was used for both training and testing groups of data. Our system had two training steps: one for the transfer learning training and the other for the random forest classifier training. We used a separate validation dataset for transfer learning training, which contained single 83 'normal' and 54 'abnormal' eardrum images 
(not pairs) during the retraining of the lookup table generation feature extraction phase. We could allocate more cases for training because we used a separate dataset for validation.

We used $\mathrm{k}$-fold $(\mathrm{k}=3)$ cross-validation to test the generalizability of our results. Because the number of 'normal-abnormal' and 'abnormal-normal' pair images was low, the fold number (k) was also kept low. The paired images were divided into three random groups for each category: one group was used for testing and the other two groups were used for training. The training group was used to learn the network parameters in transfer learning and fit a model for the tree bagger classifier part. Because the data were divided into groups before the system was run, we put the same pair in the same group, either in training or testing. Accordingly, we made sure that each patient's eardrum image pairs were used for either training or testing, but not both.

The tree bagger algorithm was also evaluated with a three-fold cross-validation method. To properly model the system, the size of the dataset and number of categories play an important role in the tree bagger classifier. We empirically decided to use five trees to model the classifier because we had 100 pairs for training and 50 pairs for testing and the number of categories was four (N-N, N-A, A-A, A-N).

\section{Results and Discussion}

We used single eardrum images to train and test the system for the transfer learning part of the training with classification accuracy as a measure for each training fold. We retrained the transfer learning for extracting the lookup table values twice: before and after adding normal-abnormal image pairs. Because the number of 'normal-abnormal' pair images was limited, we started with 'normal-normal' and 'abnormal-abnormal' pair images and with the classification categories of normal or abnormal. Subsequently, we performed the experiments adding 'normal-abnormal' eardrum pair images and compared them. Table 2 shows these two experimental results for these cases.

Table 2. Transfer learning classification accuracies for single images as normal or abnormal.

\begin{tabular}{ccccccc}
\hline & \multicolumn{2}{c}{ Before Normal-Abnormal Cases Added } & \multicolumn{3}{c}{ After Normal-Abnormal Cases Added } \\
& Training & Validation & Testing & Training & Validation & Testing \\
& $89.1 \%$ & $86.7 \%$ & $80.3 \%$ & $76.3 \%$ & $82.4 \%$ & $78.8 \%$ \\
Fold 1 & $85.4 \%$ & $93.3 \%$ & $82.9 \%$ & $86.8 \%$ & $70.6 \%$ & $78.8 \%$ \\
Fold 2 & $92.0 \%$ & $80.0 \%$ & $86.8 \%$ & $87.6 \%$ & $82.4 \%$ & $78.6 \%$ \\
Fold 3 & $88.8 \%$ & $86.7 \%$ & $83.3 \%$ & $83.6 \%$ & $78.4 \%$ & $78.7 \%$ \\
Average & $3.3 \%$ & $6.7 \%$ & $3.3 \%$ & $6.3 \%$ & $6.8 \%$ & $0.1 \%$ \\
\hline Standard Deviation & & &
\end{tabular}

Before adding normal-abnormal pairs' eardrum images, training, validation, and testing accuracies were $88.8 \% \pm 3.3 \%, 86.7 \% \pm 6.7 \%$, and $83.3 \% \pm 3.3 \%$, respectively, as seen in Table 2. However, adding normal-abnormal cases decreased the accuracies for to $83.6 \% \pm 6.3 \%, 78.4 \% \pm 6.8 \%$, and $78.7 \pm 0.1 \%$. This training step was used just for creating a lookup table and extracting lookup table features.

After creating the lookup table with transfer learning, we experimentally tested the lookup table-based feature extraction and handcrafted feature extraction (see Methodology Section). Lookup table based feature extraction was the first step of the feature extraction phase. The handcrafted features were registration errors, the number of counts in bins of the histogram of $\mathrm{L}^{*} \mathrm{a}^{*} \mathrm{~b}^{*}$, mean, and other statistical measurements (standard deviation, skewness, and kurtosis), and these were concatenated in each step, and the system was tested after each concatenation.

Figure 4 presents the system accuracies for normal-normal (N-N), abnormal-abnormal (A-A), and all pairs (N-N, A-A, N-A, and A-N). After adding N-A, A-N pair images, the accuracies decreased. Before the N-A pairs added to the dataset, the lookup table based feature extraction accuracy result was the highest accuracy with $85.1 \%$ and $7.6 \%$ low standard deviation. After adding A-N pairs, the highest accuracy result was $85.8 \% \pm 6.4 \%$ 
for concatenated registration error and histogram of $L^{*} a^{*} b^{*}$ features to the lookup table based features.
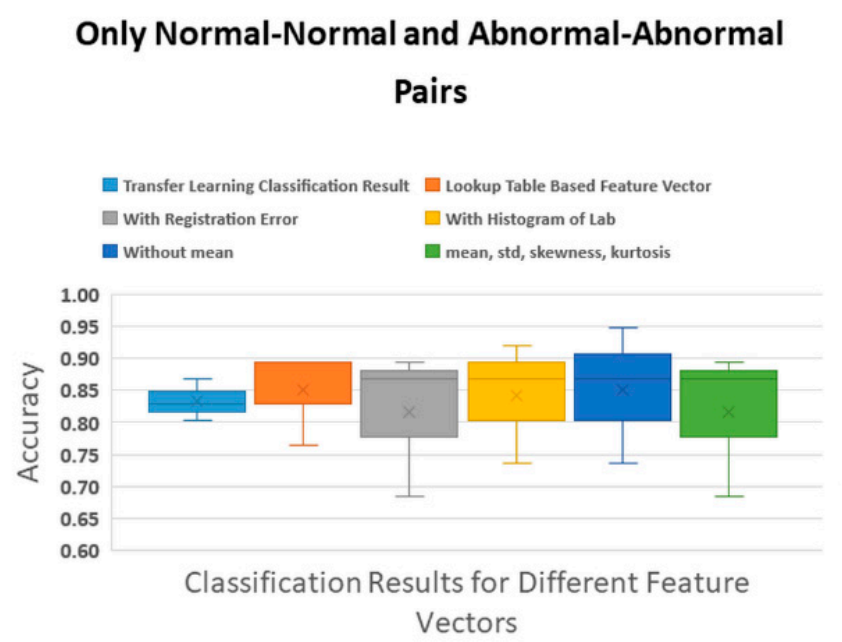

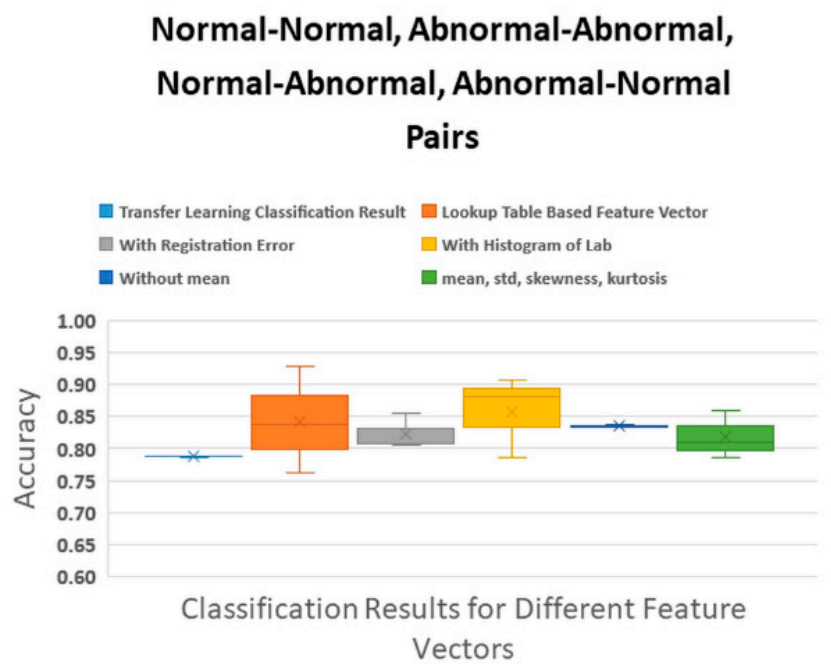

Figure 4. Results of Three-Fold Classification Accuracies for Different Feature Vectors Only Normal-Normal and AbnormalAbnormal Pairs and after added Abnormal-Normal/Normal-Abnormal Pairs.

When the paired image classification accuracies are compared with those of the single images, we observe some improvements from $83.3 \%$ (SD $\pm 3.3 \%$ ) to $85.1 \%(\mathrm{SD} \pm 7.6 \%$ ) (N-N and A-A pairs) and from 78.8\% (SD $\pm 0.1 \%)$ to $85.8(\mathrm{SD} \pm 6.4 \%)(\mathrm{N}-\mathrm{N}, \mathrm{A}-\mathrm{A}, \mathrm{N}-\mathrm{A}$, and A-N pairs). Unfortunately, most of the improvements are not statistically significant according to the $\mathrm{t}$-test between the classification results of transfer learning and each tested pair features. For the same category pair (i.e., N-N and A-A) images, the average $p$-value is 0.80 , while, for all category pair $(\mathrm{N}-\mathrm{N}, \mathrm{A}-\mathrm{A}, \mathrm{N}-\mathrm{A}$, and $\mathrm{A}-\mathrm{N})$ images, the average $p$-value is 0.16 . While the $p$-values decreased, they were not statistically significant $(<0.05)$. However, the $p$-value of the t-test between the classification results of transfer learning and all features (except for the mean value of the $L^{*} a^{*} b^{*}$ color space) is 0.0004 , which is statistically significant. The reason is standard deviations of both results for three-fold cross-validation are $0.1 \%$, and $0.2 \%$, while their accuracy values are $78.7 \%$ and $83.3 \%$, respectively. Hence, all three-fold results were consistent with just small differences.

In Figure 5, because N-N and A-A pair distributions were well separated into separate quadrants of the feature space, our expectation was the normal-abnormal pair values distributed to the other quadrants. For example, in Figure $5 \mathrm{a}, \mathrm{N}-\mathrm{N}$ pairs both right and left values less than 0 , A-A pairs values greater than -0.5 , and A-N (ear pair right side abnormal and left side normal) pairs (represented with a black circle) supposed to distribute lower-right quadrant of the figure. Likewise, for N-A (ear pair right side normal and left side abnormal) pairs (represented with blue circle) are supposed to distribute the upperleft quadrant of Figure 5a. However, black and blue circles mixed in N-N and A-A pair values are presented in Figure 5a,b. This caused a decrease in the accuracies for transfer learning test results and lookup table based feature extraction system results after adding normal-abnormal pair images.

After we observed these unexpected distributions of blue (i.e., N-A) and black circles (i.e., A-N), our ENT expert examined the normal-abnormal pair images, which were selected from video frames of both adult and pediatric patients' otoscope video clip. He labeled the normal-abnormal pair images as 'subtle' or 'not subtle', and 63.3\% (19/30) of the pair images were labeled as 'subtle'. Figure 6 illustrates four examples of pair images that were labeled as 'subtle' by our ENT specialist and misclassified by transfer learning. 


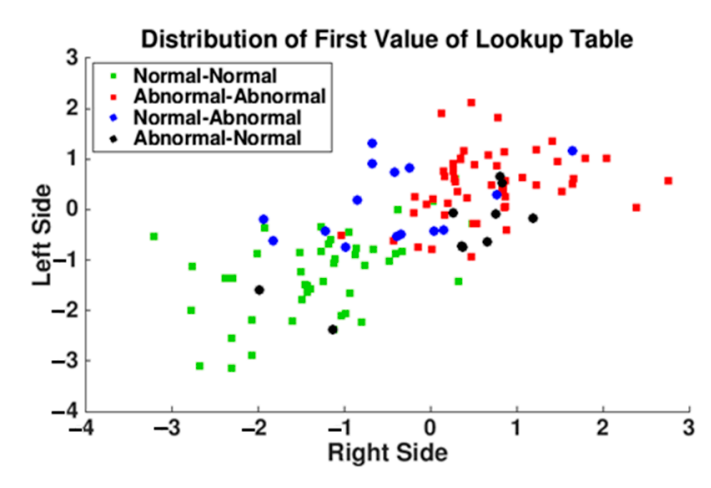

(a) Distribution of First Values of Lookup Table

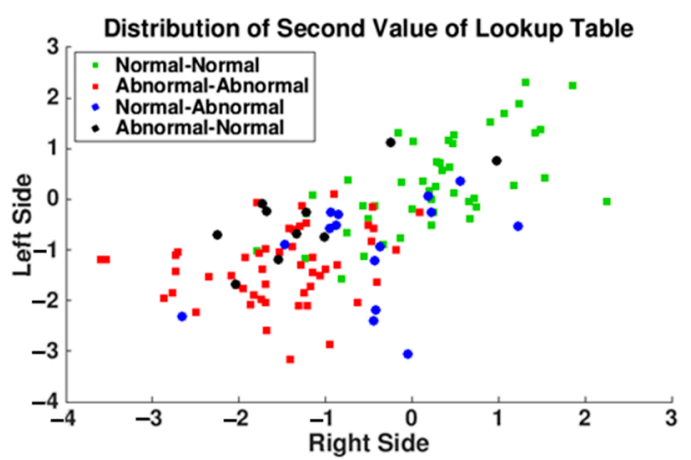

(b) Distribution of Second Values of Lookup Table

Figure 5. Lookup value distribution of Right and Left side of eardrum for Normal-Normal, Abnormal-Abnormal, NormalAbnormal, and Abnormal-Normal pairs. While Normal-Normal pairs first lookup table values located in the lower-left corner and Abnormal-Abnormal pairs first lookup table values located in the upper-right corner of (a), and vice versa for (b). The first values of right and left normal pairs (N-N) were less than zero, and for abnormal pairs (A-A), the same values were greater than -0.5 (a). For the second lookup table values, N-N and A-A pair corners switched (b). Our expectation was Normal-Abnormal pairs would state on the upper-left, and Abnormal-Normal pairs would state on the lower-right corner of (a), and vice versa for (b). However, Normal-Abnormal and Abnormal-Normal pairs stated mixed into other pairs. The number of Normal-Abnormal and Abnormal-Normal pairs are very limited, and most of them are subtle.
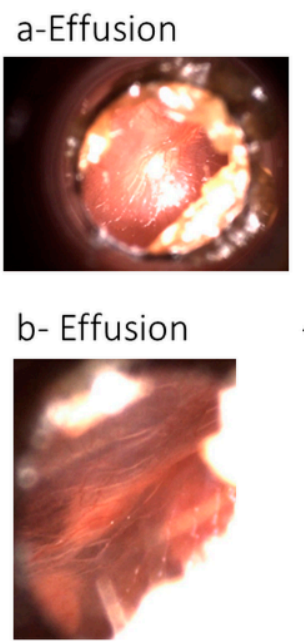
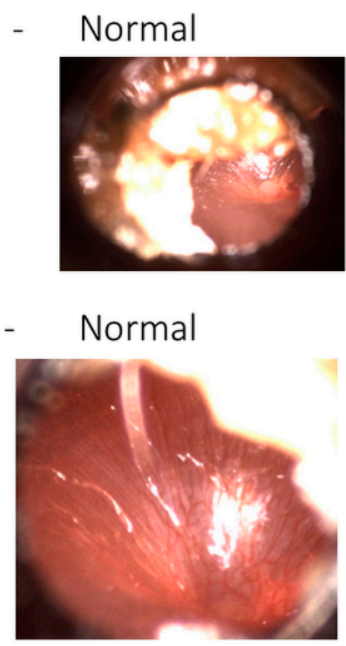

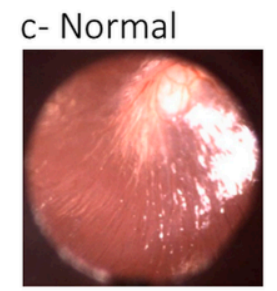

d-Effusion

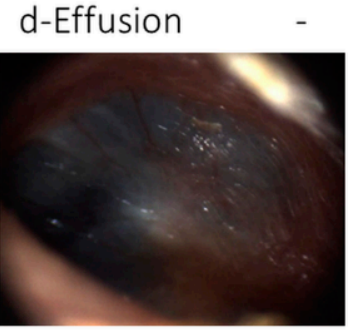

Effusion

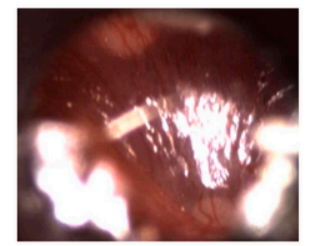

Normal

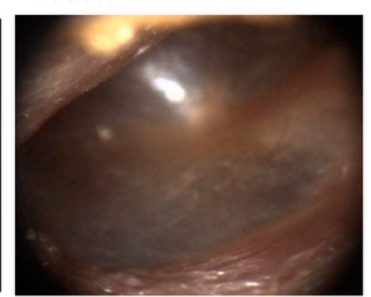

Figure 6. Examples of pair misclassified eardrum images labeled as subtle. Images are shown with the left ear on the right and right ear on the left. (a) Effusion(right ear)-Normal(left ear), (b) Effusion(right ear)-Normal(left ear), (c) Normal(right ear)-Effusion(left ear), (d) Effusion(right ear)-Normal(left ear).

Imaging problems may be the reason for misclassification. Three pairs had illumination problems that manifest themselves on images as yellow or white light spots, which makes it difficult for the camera to focus on the eardrum region. Another common problem was ear wax and hairs, because they were closer than the eardrum to the otoscope, reflecting light. Furthermore, the eardrum region of the image was not enough to classify its category. The blurry parts of the images were another problem with these images. The second column of Figure 6 demonstrates images with out of focus problems. While Figure 6c-Normal is an in-focus image, Figure 6c-Effusion is blurry between the two regions with light reflections. In contrast, Figure 6d-Effusion and d-Normal contain blurry regions, regardless of the amount of light reflection.

The accuracy of the system will likely decrease, depending on the problems (light, wax, hair, or blurring) of the normal-abnormal pairs' images. However, it should be noted that 
some of these problems (e.g., obstruction of the view by wax) also remain current barriers to traditional clinical otoscopy. Nonetheless, for this study, we wanted to experimentally test and investigate the normal-abnormal cases with a limited amount of data. While the improvements in accuracies are not statistically significant (most likely because of limited data), we still observed an increase in them using our approach, and this increase could likely be due to additional features that are extracted from the paired images and used together. Our paired image classification approach is the first for classifying pair eardrum images together, and the results are promising.

OtoPair is a novel system that classifies a pair of eardrum images together, which takes advantage of the similarities between the eardrums of an individual. This approach builds upon our previous OtoMatch content-based image retrieval (CBIR) system by creating deep learning-based lookup tables. Thus, OtoMatch sets up the infrastructure for the OtoPair system to find the similarities between a pair of eardrums. OtoPair extracts additional features, such as those that are derived from color and registration error, to classify the pair images. Additionally, OtoMatch is a content-based eardrum image retrieval system, not a classifier. OtoMatch was trained for normal, middle ear effusion, and tympanostomy tube conditions, while OtoPair was trained for 'normal' and 'abnormal' eardrum images.

\section{Conclusions}

In this study, we propose a system for classifying pair eardrum images together as 'normal-normal', 'abnormal-abnormal', 'normal-abnormal', and 'abnormal-normal'. To the best of our knowledge, this is the first study that classifies a pair of eardrum images of the same patient together. We used two feature extraction methods to classify the pair of images: deep learning-based and handcrafted, and combined the resulting features of two sides of eardrum images to classify the pair of images together. Subsequently, we analyzed the results of one side of eardrum images and pair eardrum images with and without 'normal-abnormal' and 'abnormal-normal' cases.

We also compared the results after extracting each group of features of the paired images. According to the experimental results, the highest accuracy was $85.8 \%( \pm 6.4 \%)$ for all types of pair image classification. The features of concatenated registration error and histogram of $\mathrm{L}^{*} \mathrm{a}^{*} \mathrm{~b}^{*}$ features. However, the only statistically significant result of the difference between single side eardrum image classification with transfer learning was due to all of the extracted and concatenated features (without the feature of the mean of $\mathrm{L}^{*} \mathrm{a}^{*} \mathrm{~b}^{*}$ color space) with $83.5 \%( \pm 0.2 \%)$ accuracy. Other experiments did not create any statistically significant difference. Still, at least one statistically significant result is promising with all concatenated features, except the mean of $\mathrm{L}^{*} \mathrm{a}^{*} \mathrm{~b}^{*}$ color space features.

One of the study's limitations is the small number of A-N (abnormal-normal) paired images, and the abnormal class only consists of otitis media effusion and tympanostomy tube categories. In addition to this, 63.3\% (19/30) of the existing A-N pair images were subtle as assessed by a specialist. Future studies will include a larger number of pair images for each category of eardrum pairs. We also observed that transfer learning based lookup table values for the same category pairs could be easily classified according to differently labeled pair images. Therefore, we can use the lookup table values to select subtle images and automatically eliminate them from the training dataset for future studies.

Author Contributions: Conceptualization, S.C., A.C.M., and M.N.G.; methodology, S.C., and M.N.G.; software, S.C.; validation, S.C., and M.N.G.; formal analysis, S.C., and M.N.G.; investigation, S.C.; resources, A.C.M., T.T., G.E., C.E., N.T.-S., and M.N.G.; data curation, A.C.M., T.T., G.E., C.E., and N.T.-S.; Writing-Original draft preparation, S.C.; Writing-Review and editing, S.C., A.C.M., and M.N.G.; visualization, S.C.; supervision, A.C.M., and M.N.G.; project administration, A.C.M., and M.N.G.; funding acquisition, A.C.M., and M.N.G. All authors have read and agreed to the published version of the manuscript. 
Funding: This research was funded by Award R21 DC016972 (PIs: Gurcan, Moberly) from National Institute on Deafness and Other Communication Disorders. The content is solely the responsibility of the authors and does not necessarily represent the official views of the National Institute on Deafness and Other Communication Disorders or the National Institutes of Health.

Institutional Review Board Statement: The images used in this study were captured at Ear, Nose, and Throat (ENT) and primary care clinics from both adult and pediatric patients at the Ohio State University (OSU) and Nationwide Children's Hospital (NCH) in Columbus, Ohio, US with the IRB approval (Study Number: 2016H0011). Furthermore, all the samples were fully anonymized by the rules set by the Ohio State University, Institutional Review Board.

Data Availability Statement: The data that support the findings of this study are openly available in Zenodo at https:/ / doi.org/10.5281/zenodo.4404755.

Conflicts of Interest: Computing interest: Moberly and Gurcan are directors of Otologic Technologies, Inc.

\section{References}

1. Cole, L.K. Otoscopic evaluation of the ear canal. Vet. Clin. North Am. Small Anim. Prac. 2004, 34, 397-410. [CrossRef]

2. Pichichero, M.E.; Poole, M.D. Comparison of performance by otolaryngologists, pediatricians, and general practioners on an otoendoscopic diagnostic video examination. Int. J. Pediatric Otorhinolaryngol. 2005, 69, 361-366. [CrossRef]

3. Goggin, L.S.; Eikelboom, R.H.; Atlas, M.D. Clinical decision support systems and computer-aided diagnosis in otology. Otolaryngol. Head Neck Surg. 2007, 136, s21-s26. [CrossRef]

4. Khan, M.A.; Kwon, S.; Choo, J.; Hong, S.M.; Kang, S.H.; Park, I.H. Automatic detection of tympanic membrane and middle ear infection from oto-endoscopic images via convolutional neural networks. Neural Netw. 2020, 126, 384-394. [CrossRef]

5. Kuruvilla, A.; Shaikh, N.; Hoberman, A.; Kovačević, J. Automated diagnosis of otitis media: Vocabulary and grammar. Int. J. Biomed. Imaging 2013, 2013. [CrossRef]

6. Başaran, E.; Cömert, Z.; Çelik, Y. Convolutional neural network approach for automatic tympanic membrane detection and classification. Biomed. Signal Process. Control 2020, 56, 101734. [CrossRef]

7. Kasher, M.S. Otitis Media Analysis-An Automated Feature Extraction and Image Classification System; Bachelor of Engineering Degree Programme in Electonics; Helsinki Metropolia University of Applied Sciences: Helsinki, Finland, 2018.

8. Başaran, E.; Cömert, Z.; Şengür, A.; Budak, Ü.; Çelik, Y.; Toğaçar, M. Chronic Tympanic Membrane Diagnosis based on Deep Convolutional Neural Network. In Proceedings of the 2019 4th International Conference on Computer Science and Engineering (UBMK), Samsun, Turkey, 11-15 September 2019; pp. 1-4.

9. Myburgh, H.C.; Jose, S.; Swanepoel, D.W.; Laurent, C. Towards low cost automated smartphone-and cloud-based otitis media diagnosis. Biomed. Signal Process. Control 2018, 39, 34-52. [CrossRef]

10. Monasta, L. Burden of disease caused by otitis media: Systematic review and global estimates. PLoS ONE 2012, 7, e36226. [CrossRef]

11. Cha, D.; Pae, C.; Seong, S.-B.; Choi, J.Y.; Park, H.-J. Automated diagnosis of ear disease using ensemble deep learning with a big otoendoscopy image database. Ebiomedicine 2019, 45, 606-614. [CrossRef]

12. Senaras, C. Autoscope: Automated Otoscopy Image Analysis to Diagnose Ear Pathology and Use of Clinically Motivated Eardrum Features. In Medical Imaging 2017: Computer-Aided Diagnosis; International Society for Optics and Photonics: San Diego, CA, USA, 2017; Volume 10134, p. 101341X.

13. Binol, H. Decision fusion on image analysis and tympanometry to detect eardrum abnormalities. In Medical Imaging 2020: Computer-Aided Diagnosis; International Society for Optics and Photonics: Houston, TX, USA, 2020; Volume 11314, p. 113141M.

14. Shie, C.-K.; Chang, H.-T.; Fan, F.-C.; Chen, C.-J.; Fang, T.-Y.; Wang, P.-C. A hybrid feature-based segmentation and classification system for the computer aided self-diagnosis of otitis media. In Proceedings of the 2014 36th Annual International Conference of the IEEE Engineering in Medicine and Biology Society, Chicago, IL, USA, 27-31 August 2014; pp. 4655-4658.

15. Viscaino, M.; Maass, J.C.; Delano, P.H.; Torrente, M.; Stott, C.; Cheein, F.A. Computer-aided diagnosis of external and middle ear conditions: A machine learning approach. PLOS ONE 2020, 15, e0229226. [CrossRef]

16. Seok, J.; Song, J.-J.; Koo, J.-W.; Kim, H.C.; Choi, B.Y. The semantic segmentation approach for normal and pathologic tympanic membrane using deep learning. BioRxiv 2019, 515007. [CrossRef]

17. Binol, H.; Niazi, M.K.K.; Essig, G.; Shah, J.; Mattingly, J.K.; Harris, M.S. Digital otoscopy videos versus composite images: A reader study to compare the accuracy of ENT physicians. Laryngoscope 2020. [CrossRef]

18. Binol, H.; Moberly, A.C.; Niazi, M.K.K.; Essig, G.; Shah, J.; Elmaraghy, C. SelectStitch: Automated frame segmentation and stitching to create composite images from Otoscope video clips. Appl. Sci. 2020, 10, 5894. [CrossRef]

19. Camalan, S. OtoMatch: Content-based eardrum image retrieval using deep learning. PLoS ONE 2020, 15, e0232776. [CrossRef]

20. Mironică, I.; Vertan, C.; Gheorghe, D.C. Automatic pediatric otitis detection by classification of global image features. In Proceedings of the 2011 E-Health and Bioengineering Conference (EHB), Iasi, Romania, 24-26 November 2011; pp. 1-4. 
21. Başaran, E.; Şengür, A.; Cömert, Z.; Budak, Ü.; Çelık, Y.; Velappan, S. Normal and Acute Tympanic Membrane Diagnosis based on Gray Level Co-Occurrence Matrix and Artificial Neural Networks. In Proceedings of the 2019 International Artificial Intelligence and Data Processing Symposium (IDAP), Fukuoka, Japan, 21-22 September 2019; pp. 1-6.

22. Senaras, C. Detection of eardrum abnormalities using ensemble deep learning approaches. In Proceedings of the Medical Imaging 2018: Computer-Aided Diagnosis, Houston, TX, USA, 23 April 2018; Volume 10575, p. 105751A.

23. JLee, Y.; Choi, S.-H.; Chung, J.W.J.A.S. Automated Classification of the Tympanic Membrane Using a Convolutional Neural Network. Appl. Sci. 2019, 9, 1827.

24. Brown, L.G. A survey of image registration techniques. ACM Comput. Surv. 1992, 24, 325-376. [CrossRef]

25. Mambo, S.; Hamam, Y.; van Wyk, B.; Djouani, K.; Siarry, P. A review on medical image registration techniques. World Acad. Sci. Eng. Technol. Int. J. Comput. Inf. Eng. 2018, 12, 1.

26. Huang, X.; Ren, J.; Guiraudon, G.; Boughner, D.; Peters, T.M. Rapid dynamic image registration of the beating heart for diagnosis and surgical navigation. IEEE Trans. Med. Imaging 2009, 28, 1802-1814. [CrossRef] [PubMed]

27. Miller, K. Modelling brain deformations for computer-integrated neurosurgery. Int. J. Numer. Methods Biomed. Eng. 2010, 26, 117-138. [CrossRef]

28. Strehl, N.; Tomei, S.; Rosenman, J.; Joshi, S. Large deformation 3D image registration in image-guided radiation therapy. Phys. Med. Biol. 2005, 50, 5869-5892.

29. Maksimov, D. Graph-matching based CTA. IEEE Trans. Med. Imaging 2009, 28, 1940-1954. [CrossRef]

30. Roche, A.; Pennec, X.; Malandain, G.; Ayache, N. Rigid registration of 3-D ultrasound with MR images: A new approach combining intensity and gradient information. IEEE Trans. Med. Imaging 2001, 20, 1038-1049. [CrossRef] [PubMed]

31. Thirion, J.-P. Non-rigid matching using demons. In Proceedings of the CVPR IEEE Computer Society Conference on Computer Vision and Pattern Recognition, San Francisco, CA, USA, 18-20 July 1996; pp. 245-251.

32. Keikhosravi, A.; Li, B.; Liu, Y.; Eliceiri, K.W. Intensity-based registration of bright-field and second-harmonic generation images of histopathology tissue sections. Biomed. Opt. Express 2020, 11, 160-173. [CrossRef] [PubMed]

33. Styner, M.; Brechbuhler, C.; Szckely, G.; Gerig, G. Parametric estimate of intensity inhomogeneities applied to MRI. IEEE Trans. Med. Imaging 2000, 19, 153-165. [CrossRef] [PubMed]

34. Kroon, D.-J. Multimodality Non-Rigid Demon Algorithm Image Registration. MatlabCentral. 2008. Available online: http:/ /www. mathworks.com/matlabcentral/ fileexchange/21451-multimodality-non-rigid-demon-algorithm-imageregistration (accessed on 17 August 2020).

35. Breiman, L. Bagging predictors. Mach. Learn. 1996, 24, 123-140. [CrossRef]

36. Matthew, W. Bias of the Random Forest out-of-bag (OOB) error for certain input parameters. Open J. Stat. 2011, 2011. [CrossRef]

37. James, G.; Witten, D.; Hastie, T.; Tibshirani, R. An Introduction to Statistical Learning; Springer: Berlin/Heidelberg, Germany, 2013. 